\title{
CMR measurements of myocardial infarct heterogeneity using MCLE and IR-FGRE: correlation with arrhythmia inducibility and severe ICD events in patients with ischemic heart disease
}

\author{
Yuesong Yang ${ }^{1 *}$, Kim A Connelly ${ }^{2}$, Tawfiq Zeidan', Subodh B Joshi ${ }^{2}$, John J Graham², Gideon A Paul', \\ Rhonda Walcarius ${ }^{1}$, Alexander J Dick ${ }^{3}$, Eugene Crystal ${ }^{1}$, Graham Wright ${ }^{1}$
}

From 15th Annual SCMR Scientific Sessions

Orlando, FL, USA. 2-5 February 2012

\section{Summary}

This study used CMR to evaluate patients with IHD prior to ICD implantation and correlated CMR measurements to VA inducibility and spontaneous VA events during follow-up. The results demonstrated that the gray-zone measurement using MCLE may be more sensitive in predicting appropriate ICD therapy for VA.

\section{Background}

In addition to measures of left ventricular ejection fraction (LVEF) and clinical staging of heart failure, myocardial infarct (MI) heterogeneity including $\mathrm{MI}$ and periinfarct gray-zone (GZ) has the potential to predict the occurrence of inducible sustained ventricular arrhythmia (VA) and spontaneous VA events after implantation of implantable defibrillator (ICD) in patients with ischemic heart disease (IHD). Late-gadolinium (Gd)-enhancement (LGE) cardiac MR (CMR) using inversion-recovery fastgradient-echo (IR-FGRE) is commonly used for the determination of infarct heterogeneity in these patients. Recently, a multi-contrast late-enhancement (MCLE) sequence has been developed for better infarct heterogeneity quantification. Compared to IR-FGRE, we hypothesized that MCLE may be a more sensitive method to predict the occurrence of inducible VA and severe events post-ICD implantation.

${ }^{1}$ Imaging Research and Cardiology, Sunnybrook Health Sciences Centre Toronto, ON, Canada

Full list of author information is available at the end of the article

\section{Methods}

This study used CMR to evaluate patients with IHD prior to ICD implantation and correlated CMR measurements to VA inducibility and spontaneous VA events during follow-up. The MRI protocol included LV functional parameter assessment using steady-state free precession (SSFP), as well as LGE-MRI using IR-FGRE and MCLE post double-dose Gd injection. LV functional parameters were measured using Q-Mass or CMR42 software. The GZ analysis in IR-FGRE used a full-width half-maximum method. For MCLE, GZ analysis used a semi-automated data clustering algorithm. An unpaired $t$-test with unequal variance was used for the statistical analysis of the proportion of GZ, MI core and total MI relative to LV myocardium mass.

\section{Results}

Twenty-five patients with IHD for planned ICD implantation (age 64 +/ - 10 yrs, 88\% men, average LVEF 26.2 $+/-10.4 \%$, 40\% secondary prevention) were enrolled. All patients completed the MRI protocol, ventricular electro-physiology study (EPS), and six months follow-up at the ICD clinic. Eight patients (32\%) had inducible VA during programmed ventricular stimulation and six of these eight patients had at least one appropriate shock for VF from the ICD at follow-up. Another two patients without inducible VA had appropriate shocks from the ICD. CMR measurements of LVEF and myocardial infarct heterogeneity are shown in Tables $1 \& 1(2)$. LVEF 


\begin{tabular}{|c|c|c|c|c|c|c|}
\hline & \multicolumn{2}{|c|}{ Inducible VA $(n=8)$} & \multicolumn{2}{|c|}{ Non-inducible VA $(n=17)$} & \multicolumn{2}{|c|}{$P$ value } \\
\hline & MCLE & IR-FGRE & MCLE & IR-FGRE & MCLE & IR-FGRE \\
\hline GZIVM (\%) & $15.2 \pm 2.7$ & $15.6 \pm 3.8$ & $11.9 \pm 5.1$ & $10.6 \pm 5.2$ & 0.04 & 0.01 \\
\hline MI core/LVM (\%) & $28.3 \pm 7.1$ & $25.3 \pm 9.6$ & $19.9 \pm 11.3$ & $16.8 \pm 10.2$ & 0.04 & 0.06 \\
\hline Total MI/LVM (\%) & $43.4 \pm 6.9$ & $40.9 \pm 13.2$ & $31.9 \pm 15.2$ & $27.3 \pm 15.2$ & 0.02 & 0.04 \\
\hline LVEF (\%) & \multicolumn{2}{|c|}{$18.0 \pm 8.2$} & \multicolumn{2}{|c|}{$30.1 \pm 9.2$} & \multicolumn{2}{|c|}{0.005} \\
\hline & \multicolumn{2}{|c|}{ With shocks for VA $(n=8)$} & \multicolumn{2}{|c|}{ Without shocks for VA (n=17) } & \multicolumn{2}{|c|}{$P$ value } \\
\hline & MCLE & IR-FGRE & MCLE & IR-FGRE & MCLE & IR-FGRE \\
\hline GZIVM (\%) & $15.3 \pm 2.7$ & $14.1 \pm 4.4$ & $11.8 \pm 5.1$ & $11.3 \pm 5.5$ & 0.04 & 0.19 \\
\hline MI core/LVM (\%) & $26.3 \pm 11.1$ & $22.9 \pm 9.5$ & $20.8 \pm 10.5$ & $17.9 \pm 11.0$ & 0.26 & 0.26 \\
\hline Total MI/LVM (\%) & $41.6 \pm 10.4$ & $36.9 \pm 13.6$ & $32.8 \pm 15.0$ & $29.2 \pm 16.3$ & 0.10 & 0.23 \\
\hline LVEF (\%) & \multicolumn{2}{|c|}{$21.9 \pm 7.7$} & \multicolumn{2}{|c|}{$28.3 \pm 11.1$} & \multicolumn{2}{|c|}{0.11} \\
\hline
\end{tabular}

Figure 1

and GZ measurements from IR-FGRE and MCLE showed a statistically significant difference between groups of inducible and non-inducible VA; however, only the proportion of GZ measured with MCLE demonstrated a statistical difference $(\mathrm{P}=0.04)$ between patients with and without ICD shocks for VA.

\section{Conclusions}

CMR measurements of myocardial heterogeneity using MCLE and IR-FGRE are good predictors for the occurrence of inducible VA; however, the gray-zone measurement using MCLE may be more sensitive in predicting appropriate ICD therapy for VA.

\section{Funding}

CIHR.

\section{Author details}

${ }^{1}$ Imaging Research and Cardiology, Sunnybrook Health Sciences Centre,

Toronto, ON, Canada. ${ }^{2}$ Cardiology, St. Michael Hospital, Toronto, ON, Canada.

${ }^{3}$ Cardiology, Ottawa Heart Institute, Ottawa, ON, Canada.

Published: 1 February 2012

doi:10.1186/1532-429X-14-S1-0105

Cite this article as: Yang et al:: CMR measurements of myocardial infarct heterogeneity using MCLE and IR-FGRE: correlation with arrhythmia inducibility and severe ICD events in patients with ischemic heart disease. Journal of Cardiovascular Magnetic Resonance 2012 14(Suppl 1): 0105.
Submit your next manuscript to BioMed Central and take full advantage of:

- Convenient online submission

- Thorough peer review

- No space constraints or color figure charges

- Immediate publication on acceptance

- Inclusion in PubMed, CAS, Scopus and Google Scholar

- Research which is freely available for redistribution

Submit your manuscript at www.biomedcentral.com/submit 